Meta

Journal des traducteurs

Translators' Journal

\title{
Pour une théorie de la traduction inspirée de sa pratique
}

\section{Danica Seleskovitch}

Volume 25, numéro 4, décembre 1980

URI : https://id.erudit.org/iderudit/004084ar

DOI : https://doi.org/10.7202/004084ar

Aller au sommaire du numéro

Éditeur(s)

Les Presses de l'Université de Montréal

ISSN

0026-0452 (imprimé)

1492-1421 (numérique)

Découvrir la revue

Citer cet article

Seleskovitch, D. (1980). Pour une théorie de la traduction inspirée de sa pratique. Meta, 25(4), 401-408. https://doi.org/10.7202/004084ar d'utilisation que vous pouvez consulter en ligne.

https://apropos.erudit.org/fr/usagers/politique-dutilisation/ 


\title{
Pour une théorie de la traduction inspirée de sa pratique
}

\author{
D. Seleskovitch
}

La première question qu'il convient de se poser à un colloque réuni pour débattre des services que la théorie peut apporter à la pratique est de savoir s'il existe réellement deux champs d'action nettement délimités dans lesquels s'isoleraient respectivement les théoriciens et les praticiens, la distinction impliquant que les seconds ne sauraient pas théoriser alors que les premiers ne sauraient que théoriser. Je ne crois pas que cela soit le cas. Un examen attentif du travail du praticien montre en tout cas que celui-ci témoigne d'un degré considérable de théorisation.

\section{La réussite pragmatique, point de départ de la théorisation}

Pour le praticien, la théorisation consiste le plus souvent à observer ses succès, à découvrir intuitivement ce qui les implique puis à généraliser les modalités de réussite de l'acte en éliminant peu à peu les causes d'échec. C'est cette itération, cette démarche par approches successives, que les Américains appellent «trial and error». On m'objectera qu'il s'agit là d'une pragmatique et non d'une approche théorique. Erreur; établir une systématique qui mène toujours à la réussite est une théorisation à laquelle il ne manque que l'explicitation. La réussite suppose la découverte des principes qui sont en cause, même si leur formulation fait encore défaut.

J'ai connu un cas extrême de systématisation qui n'atteignait pas le plan de la formulation verbale : au Texas, dans une station expérimentale de culture de l'arachide, on m'a montré un jour un homme qui triait très rapidement des arachides et en faisait deux tas; chacun de ces tas correspondait à une variété distincte dont l'apparence ne présentait cependant aucune différence avec l'autre. Aucun procédé scientifique n'avait permis de découvrir ce qui distinguait les graines. La forme, la couleur, le poids, le grain de la cacahuète, son degré hygrométrique avaient été trouvés semblables dans les deux variétés que seule différenciait leur végétation. Mon planteur les distinguait nettement mais était cependant incapable d'exposer les critères selon lesquels il triait.

Par cette anecdote, j'entends dire que toute abstraction qui, à partir des données de l'expérience, permet d'agir en obtenant dans des circonstances analogues des résultats analogues est déjà une théorie. Elle n'est pas encore formulée, mais elle pose les fondements de l'étape d'explicitation. 
Pour le traducteur, pour l'interprète que je suis, c'est à la réussite de l'opération de traduction qu'il convient d'accrocher la réflexion théorique. Prendre un autre point de départ ne peut à nos yeux mener qu'à des spéculations à vide.

\section{Peut-on théoriser en faisant abstraction de la pratique?}

Claude Bernard, parlant en médecin praticien, disait de la théorie qu'elle était «l'hypothèse vérifiée après qu'elle ait été soumise au contrôle du raisonnement et de la critique expérimentale». Il l'opposait à la doctrine qui «procède par affirmation et par déduction purement logique».

Pour théoriser sur la traduction il faut, si l'on est d'accord avec Claude Bernard, poser d'abord une hypothèse d'explication du processus de la traduction, puis la soumettre au contrôle du raisonnement et de la critique expérimentale.

Le reproche essentiel que le praticien peut faire aux théoriciens qui font abstraction de la pratique est que, laissant inexprimée leur hypothèse de départ, ils sont dans l'incapacité de la vérifier. Je prendrai à nouveau à titre d'exemple une anecdote véridique. Une école d'interprétation et de traduction d'une université européenne a mis au point un test d'aptitude à la traduction sans formuler explicitement d'hypothèse sur la nature de l'opération pour laquelle il s'agit de définir une aptitude. Le test consistait à montrer aux candidats une grille de correspondances entre lettres et nombres telle que ci-dessous :

\begin{tabular}{cccccccccccc}
\multicolumn{1}{c}{ Grille de correspondance } \\
$\mathrm{a}$ & $\mathrm{b}$ & $\mathrm{c}$ & $\mathrm{d}$ & $\mathrm{e}$ & $\mathrm{f}$ & $\mathrm{g}$ & $\mathrm{h}$ & $\mathrm{i}$ & $\ldots$...etc. \\
\hline 3 & 8 & 2 & 5 & 4 & 1 & 7 & 6 & 9 & $\ldots$...etc.
\end{tabular}

Les lettres sont présentées dans l'ordre alphabétique, les nombres dans le désordre. Après qu'ils aient appris la grille par cœur, les candidats recevaient des séries de chiffres dans le désordre, telles que: $7,4,2,14,5,23,4$ etc., et il leur fallait placer les chiffres sous les lettres correspondantes. Étaient considérés aptes à la traduction ceux qui avaient, dans le temps le plus court, établi le plus grand nombre de correspondances exactes. Ce test était censé mesurer la «vitesse d'apprentissage» - en clair: la vitesse d'apprentissage d'équivalences entre langues qu'il suffirait, une fois apprises, d'appliquer pour traduire. Il y avait là une hypothèse de départ dont l'absurdité n'apparaissait pas prima facie du fait qu'elle était laissée à l'état implicite : traduire, ce serait appliquer des équivalences et apprendre à traduire, apprendre des équivalences. Cette école affirmait une doctrine mais de façon implicite.

Nous avons là deux exemples poussés à l'extrême, d'une part une pratique qui ne sait pas s'expliquer sur ce qu'elle sait faire, d'autre part une doctrine si fermement ancrée qu'elle ne songe pas à se remettre en cause en s'affirmant explicitement. 
Pour en revenir à la traduction, il existe effectivement deux champs d'action distincts: le premier où œuvrent depuis des milliers d'années des praticiens qui n'expliquent pas toujours ce qu'ils font ou ne l'expliquent pas de façon convaincante, et le second où œuvrent, essentiellement depuis quelques décennies, des théoriciens qui ne formulent pas toujours leurs hypothèses de départ et qui les soumettent moins souvent encore à la vérification expérimentale.

\section{Les doctrines linguistiques de la traduction}

$\mathrm{Si}$, en ouvrant un livre ou un journal, en entendant un discours ou une conversation, nous pensions n'y trouver que du français ou de l'anglais, de l'espagnol ou du chinois, nous poserions l'hypothèse implicite que la seule connaissance que nous mettons en cuvre lorsque nous lisons ou écrivons, parlons ou entendons parler, est la connaissance de la langue dans laquelle sont composés ces textes ou ces discours.

C'est l'hypothèse implicite posée par les théories linguistiques de la traduction. En plein essor depuis Saussure, la linguistique synchronique s'est donné une assise scientifique en dissociant l'étude des langues et de leur fonctionnement de celle de leur emploi (dichotomie langue-parole). Puis à côté d'un approfondissement des travaux sur les mécanismes et le fonctionnement du langage au niveau de la langue, on a vu au cours des trente dernières années se développer la psycholinguistique, la sociolinguistique, les théories de la communication, les recherches empiriques sur les actes de parole et sur les structures de la conversation, sans parler de la linguistique des textes qui étudie les structures transphrastiques de la langue. Toutes ces études dépassent largement le territoire assigné à la langue par la linguistique post-saussurienne, sans pour autant se départir de leur caractère scientifique.

En revanche, des structuralistes comme G. Mounin ou R. Jakobson qui ont traité de la traduction humaine sous l'angle du seul fonctionnement de la langue, un générativiste comme $\mathbf{N}$. Chomsky qui a cuvré indirectement pour la machine à traduire, n'ont pas su voir que pour étudier la traduction, il faut quitter le domaine des systèmes de signes articulés, le domaine de la compétence linguistique neutre d'un "native speaker», pour pénétrer dans celui de l'acte de communication qui est à la fois actualisation de la langue et expression d'une pensée individuelle, le domaine des messages dont la parole est le porteur et qui sont à la fois composés de langue et de contenus cognitifs qui ne s'attachent que fugitivement aux signes linguistiques. L'étude de la traduction exige que soient pris en considération non seulement la compétence linguistique du sujet comprenant et parlant, mais aussi son bagage cognitif et ses capacités logiques.

En se contentant de comparer les langues, les doctrines linguistiques de la traduction analysent le résultat de la traduction et non la traduction ellemême; celle-ci, obligatoirement, précède toute possibilité de comparaison (cf. Delisle 1980). La comparaison est postérieure à l'acte qui la rend possible; à ne voir que son résultat, on ne voit que les deux langues en présence, ce qu'on a appelé la langue de départ et la langue d'arrivée, et il était tentant de conclure 
de la matière linguistique ainsi observée à la convertibilité de cette matière et d'affirmer implicitement que traduire, c'est convertir une langue en une autre.

Le grand reproche que l'on peut faire aux doctrines linguistiques de la traduction est de ne pas avoir formulé explicitement cette affirmation qui est leur hypothèse de base, la soustrayant ainsi à la critique du raisonnement et à la vérification expérimentale.

La langue de départ n'est pas le point de départ, la langue d'arrivée n'est pas le point d'aboutissement de l'opération que réalise le traducteur. Celui-ci ne perçoit au départ que des contrastes de noir et blanc sur le papier, qu'il interprète à des degrés variables selon ses connaissances: s'il ne connaît que l'alphabet d'une langue (grec ou cyrillique par exemple) il n'y verra que des caractères d'imprimerie, si on lui présente des énoncés hors contexte et hors situation, il n'y verra que les significations que sa connaissance des langues lui permettra de dégager; en revanche, en situation de communication il y trouvera le sens des messages que leur auteur veut transmettre.

\section{La théorie interprétative de la traduction}

\section{Le sens}

L'avènement du magnétophone, l'invention des bandes magnétiques permet aujourd'hui l'observation seconde par seconde de la traduction orale (ou interprétation), ouvrant la voie à une étude scientifique du processus de la traduction. Les interprètes de mon équipe de l'ESIT : Karla Déjean, Mariano Garcia-Landa, Marianne Lederer en ont fait abondamment usage, multipliant les expériences et les observations pragmatiques et sortant la traduction de la seule comparaison des langues en présence. Parallèlement deux chercheurs canadiens, Jean Delisle à l'Université d'Ottawa et Denis Juhel à Fredericton et maintenant Laval, dégageaient de leur enseignement une optique identique. Qu'il s'agisse de traduction écrite ou d'interprétation orale, les conclusions auxquelles nous arrivons sont les mêmes : comprendre un texte ou un discours ne consiste pas seulement à identifier les contenus sémantiques permanents des signes linguistiques et à saisir la signification qui se dégage de leur combinaison syntaxique en phrases, mais aussi à discerner les éléments cognitifs autres que linguistiques qui, en une situation donnée, s'attachent à l'énoncé.

Cette double composante de la compréhension se fond en un élément unique : le sens du dire. À la lecture d'un texte ou à l'audition d'un discours, le traducteur qui partage, au même titre que le destinataire du message, les connaissances supposées chez celui-ci par l'auteur, dégage le sens en une synthèse immédiate des éléments sensibles et des éléments cognitifs en présence. Pour "traduire», il peut dès lors utiliser toutes les ressources de sa langue maternelle car il n'est plus entravé par l'original.

L'observation de la pratique ayant permis de poser l'hypothèse que traduire consiste à comprendre un texte ou un discours puis à faire un texte ou un discours qui ait le même contenu dans une autre langue, il s'agissait de vérifier, en s'appuyant sur des traductions réussies, en quoi consistaient les adjonctions 
cognitives qui complétaient le sémantisme des mots et des phrases, et où s'arrêtait le sens par rapport à l'infini de l'univers conceptuel.

Le cadre de cette communication ne me permet pas d'entrer dans le détail des analyses publiées ailleurs (Danica Seleskovitch, 1975, 1976, 1978; Marianne Lederer, 1976, 1980; Jean Delisle 1980), qu'il me suffise de dire que l'interprétation de conférence, que je pratique depuis 30 ans maintenant, m'offrait non seulement une grande facilité expérimentale mais présentait en outre l'immense avantage de correspondre à une situation d'énonciation où toutes les composantes de la communication sont présentes et dans laquelle l'on peut constater sur le champ que, si la traduction ne les prend pas en compte, le dialogue des interlocuteurs s'interrompt ou se fourvoie. Une analyse fine de l'interprétation consécutive puis simultanée $m$ 'a permis de délimiter le sens, d'une part par rapport aux contenus sémantiques de la langue, et d'autre part par rapport aux implications que comporte tout échange verbal, inférences et sous-entendus qui se greffent sur le sens mais restent inexprimés. La traduction situe ainsi son domaine entre celui de la sémantique et celui de l'exégèse, elle n'est ni transmutation des langues ni glose.

Avant d'examiner les éléments qui différencient le sens réexprimé par la traduction, des significations attachées en permanence aux mots et aux phrases, donnons un exemple rapide des frontières entre la traduction et la glose :

Le discours qui dirait : «le chat est dehors", n'exprime pas seulement son sens (le mot chat désigne en l'occurrence ma chatte noire qui est petite et nerveuse, qui est tombée un jour du $5^{e}$ étage dans la rue, etc., le mot dehors désigne le bout de jardin qui se trouve derrière la cuisine de notre pavillon de Bourg la Reine, etc.), ce discours possède aussi des implications qui ne relèvent plus du dire du locuteur mais de l'intention de sa communication. Les paroles «le chat est dehors» impliquaient: «il faut le faire rentrer». En effet, ma chatte avait à ce moment-là une blessure à la patte et il avait été entendu qu'on l'empêcherait de sortir pendant quelques jours; il était d'autant plus important de la faire rentrer qu'il pleuvait et que le jardin était boueux...

La traduction s'en tient au sens de «le chat est dehors»; exprimer l'implication «il faut le faire rentrer» serait une glose qui transgresserait le dire de l'auteur du message, et non une traduction qui le respecte. Mais respecter le dire ne consiste pas pour autant à s'en tenir à la signification hors contexte des mots. Ici les mots le chat ont strictement le même sens que «ma chatte noire», le mot dehors le même sens que «dans le jardin»; si les exigences idiomatiques d'une autre langue l'imposaient, «le chat est dehors» aurait pu être traduit par «la chatte noire est dans le jardin»; le sens du dire original aurait été strictement respecté. En effet si le traducteur ne se confond pas avec un exégète, il ne se confond pas non plus avec un sémanticien: les éléments sémantiques ne rendent pas compte du sens du discours, qui n'est compris qu'en fonction des éléments cognitifs qui s'y attachent de façon ad hoc. 


\section{Les compléments cognitifs}

Nous avons baptisé compléments cognitifs les éléments qui contribuent au sens du discours; ce sont: les interlocuteurs, la situation qu'ils partagent, le moment où ils se parlent, l'endroit où ils se trouvent, les connaissances qu'ils possèdent les uns et les autres et le souvenir qu'ils gardent de ce qui vient d'être dit. Ces éléments, qui s'adjoignent aux mots et aux phrases sans s'y fixer de façon définitive et donc sans devenir partie intégrante des langues, sont pris en compte intuitivement dans chaque échange verbal.

La prise en compte de celui qui parle apporte au traducteur un élément cognitif qui teinte en toutes circonstances le contenu sémantique des paroles. La traduction, en apporte la démonstration. Prenons à titre d'exemple, l'expression russe «S vami Bog» (littéralement : «avec vous, Dieu»); imaginons qu'elle soit utilisée une fois par un dirigeant soviétique, une autre fois par un dignitaire de l'église catholique, et voyons ce qu'en donnerait la traduction dans les deux cas. Nous constaterons qu'au lieu d'appliquer des équivalences pré-établies, nous tiendrons compte du sens de l'expression, c'est-à-dire à la fois du contenu sémantique des signes linguistiques et de celui qui les émet. En quittant Bonn il y a une quinzaine d'années après avoir rétabli entre l'URSS et l'Allemagne de l'Ouest un contact rompu depuis 20 ans, Khroutchev prononça en guise d'adieu les mots « $\mathrm{S}$ vami $B o g$; l'interprète les traduisit correctement en allemand en disant «Alles Gute» (Bonne chance! Good Luck!) provoquant des commentaires dans la presse allemande, qui aurait préféré une traduction plus littérale sans se rendre compte qu'elle aurait impliqué une révision déchirante de l'idéologie des dirigeants soviétiques! En juin 1979, le Pape Jean-Paul II achevait sa visite en Pologne par les mêmes mots en polonais. Il n'avait pas besoin d'interprète entre lui et la foule, mais peut-on imaginer que l'expression, identique dans sa forme linguistique à celle employée par Kroutchev, ait pu être traduite dans quelque langue que ce soit en omettant la notion de Dieu? Le sens de l'énoncé est différent dans les deux cas, dans «S vami Bog» Khroutchev mettait une amabilité, le pape une bénédiction.

Si les langues exprimaient le même sens par les mêmes contenus sémantiques, le traducteur pourrait se contenter de traduire les langues. En montrant que cela n'est pas le cas, sa pratique révèle la différence entre les significations strictement linguistiques des paroles échangées et le sens qu'elles prennent en situation.

Voyons d'autres transformations en sens de la signification des mots et des phrases. Ici prononcé au colloque de Toronto aurait été compris comme désignant le Old Dining Hall de Glendon College, aujourd'hui le 23 mai 1980, vous les participants réunis dans cette salle, etc. Ce n'est pas tout. L'orateur adapte quantitativement son discours aux connaissances permanentes qu'il suppute chez son interlocuteur; l'implicite est véhiculé avec l'explicite, constituant un complément cognitif qui fusionne à son tour en un sens pour le destinataire initié. Il y a quelques mois un haut fonctionnaire coréen déclarait littéralement dans sa langue : «Depuis les événements du 26 octobre... » L'interprète coréenne traduisit : «Depuis le 26 octobre, date de l'assassinat du Général Park...» 
En coréen et en français le sens des paroles était identique, mais en coréen, l'expression postulait la présence d'un complément cognitif qu'en français elle donnait. La proportion d'explicite et d'implicite varie constamment dans la communication en fonction du savoir partagé par les interlocuteurs; on voit les traductions en tenir compte, rendant explicite un implicite dont l'absence empêcherait la constitution du sens, supprimant aussi bien un explicite qui serait redondant. Aucun interprète n'a jamais traduit pour un Français le «Paris, France» américain autrement que par «Paris», et on est choqué lorsque la traduction ne reflète pas les mécanismes du langage, comme celle-ci : «C'est à Paris que siégeaient les principaux organes du gouvernement, le Parlement et la Chambre des Comptes qui correspondait approximativement à l'Échiquier anglais ${ }^{1}$ ", en exprimant ce qui aurait dû devenir implicite, la traduction fournit un renseignement déroutant pour le lecteur français.

Le complément cognitif constitué par l'implicite véhiculé avec l'explicite ne s'explique pas seulement par des connaissances partagées en permanence; le discours suit le fil des associations d'idées de l'orateur et poursuit un objectif rapidement discerné par ceux à qui il s'adresse, chaque argument étant compris en fonction des arguments qui l'ont précédé. Au bagage cognitif permanent s'ajoutent ainsi les éléments cognitifs apportés par le discours lui-même et constituant des compléments cognitifs que seuls peuvent saisir ceux qui l'ont suivi de bout en bout. Le traducteur en prend conscience, à qui l'on donne parfois à traduire des segments de texte dont il ne connaît pas le début!

Benveniste a parlé de signes vides : les pronoms, les déictiques sont selon lui des signes qui ont bien une fonction grammaticale mais ne se remplissent sémantiquement qu'en situation de parole. La pratique de la traduction apporte à la théorie du langage l'observation qu'en fait aucun signe linguistique n'est plein et que seule la situation de parole le remplit, donnant aux paroles un sens inédit, ad hoc, car jamais ne se cristallise sur les mêmes paroles un assortissement identique de compléments cognitifs.

Les compléments cognitifs permettent de découvrir le rapport qui associe dans le discours des signifiants inchangés et des sens toujours renouvelés - et c'est à partir du sens ainsi éclairé que le traducteur trouve l'expression idoine dans l'autre langue.

Voilà qui explique qu'à quelques exceptions près, la traduction est création dans l'autre langue et non application d'équivalences préétablies, offrant à l'étude $\mathrm{du}$ fonctionnement de la communication humaine un poste d'observation sans égal.

Dissociant intégralement les deux langues, la traduction peut respecter les contraintes imposées par le génie de la langue d'expression. La Stylistique comparée du français et de l'anglais de Vinay et Darbelnet et la théorie interprétative de la traduction que j'expose ici sont, on le voit, complémentaires. La Stylistique souligne l'importance pour l'intelligibilité de la traduction du respect de la

1. Cet exemple est tiré d'une traduction (p. 25) d'autre part en tous points remarquable du Louis XI de Paul Murray Kendal. 
langue d'expression, la théorie interprétative affirme que l'objet à exprimer ne se dégage pas seulement de la sémantique étroite de la langue mais bien de l'ensemble des éléments cognitifs qui fusionnent avec la partie stable des significations linguistiques, et que la langue d'expression retrouve tous ses droits à partir du moment où le sens est dégagé.

\section{Les difficultés de la pratique et la théorie}

En exposant la théorie interprétative de la traduction, j'ai amalgamé l'oral et l'écrit, l'interprétation et la traduction. L'expérience montre en effet qu'à condition que tous les facteurs de la chaîne de communication soient présents, le processus est le même dans les deux cas; devant le sens, traducteurs et interprètes réagissent de façon identique. Mais l'écrit présente une infinie variété de cas que l'oral ignore. Il ne s'agit pas ici du genre des textes - l'oral a ses discours poétiques ou vulgaires, techniques ou philosophiques, etc., mais il connaît toujours les mêmes circonstances d'émission et de réception. L'écrit en revanche, rémanent par définition, défie les siècles et les kilomètres de sorte qu'on ne sait pas toujours dans quelles circonstances un texte a été produit, que l'on ignore les événements auxquels il se réfère ou la matière dont il traite. Quand les compléments cognitifs commencent à faire défaut, le sens des paroles se rétrécit comme une peau de chagrin, jusqu'à atteindre le point zéro avec l'ignorance complète de tout signifié de la langue. Tous les stades intermédiaires existent en traduction pour des raisons diverses : textes anciens, civilisations aux mœurs différentes, textes techniques à la traduction desquels s'attaquent les diplômés de nos filières de littérature, etc. Alors manquent les connaissances extra-linguistiques qui leur donneraient du sens. Ce sont là des carences souvent dues à une mauvaise conception des processus de la traduction, inéluctables par contre lorsque l'accès aux informations complémentaires est impossible. Les problèmes pratiques que pose la traduction de textes que le traducteur n'appréhende pas comme un lecteur normal sont considérables, parfois insurmontables mais, si graves qu'ils soient, ce ne sont pas des problèmes théoriques. La traduction est un acte de parole; pour en dégager le processus, il faut l'étudier dans son intégrité en présence de tous les éléments qui interviennent en situation normale de communication. Pas plus qu'il ne faudrait théoriser sur la marche en étudiant un paraplégique dans son fauteuil roulant, il ne faut théoriser sur la traduction à partir des difficultés qui s'opposent à sa réalisation pratique ; sinon la pratique devient mauvaise inspiratrice de la théorie. 